\title{
Estudio de las características reproductivas de híbridos de clavel (Dianthus caryophyllus)
}

\section{Study of the reproductive characteristics of carnation hybrids (Dianthus caryophyllus)}

\author{
Dian Marcela Ríos ${ }^{1}$ y Juan José Filgueira² \\ Recibido para publicación: Septiembre 7 de 2018 - Aceptado para publicación: Noviembre 23 de 2018
}

\begin{abstract}
RESUMEN
El clavel es la principal flor de exportación de Colombia y sus principales destinos son los Estados Unidos y Europa. Colombia, es actualmente el primer productor de clavel en el mundo. Las enfermedades vasculares producidas por hongos son la causa más importante de pérdidas en este cultivo y el método de control más eficiente es contar con variedades resistentes. El reconocimiento de las características reproductivas de híbridos de clavel es el objetivo principal de este estudio que permitió en un plazo de tiempo prudente establecer un programa de producción de nuevas variedades de clavel en Colombia. Para ello se utilizaron híbridos de tres generaciones sucesivas obtenidos por autopolinización y se estudiaron las características reproductivas tanto del polen como de los óvulos, así como la cantidad de las diferentes estructuras de reproducción. Los resultados indican que tanto para los órganos de reproducción masculinos como los femeninos, existe una gran variabilidad en número de estructuras, donde la viabilidad reproductiva no está ligada a la generación endogámica. De la misma forma, la cantidad de polen no está directamente vinculada con el número de semillas producidas, así como tampoco con el número de óvulos por gineceo. Por el contrario, la cantidad de semillas si está relacionada con el número de óvulos viables por flor. La fertilidad de los híbridos expresada en términos de granos de polen vs óvulos fecundados y semillas producidas por tallo floral muestra que existe una variabilidad muy amplia en las características de los factores que determinan aspectos reproductivos de los híbridos.
\end{abstract}

Palabras clave: Mejoramiento, híbridos, órganos reproductivos de plantas.

\begin{abstract}
Carnation is the most important flower in the exportation and the principal destination are United States of America and Europe and the country is the first producer of carnation in the word. The vascular diseases are the most important cause of losses in the crop and the most efficient method of control is to count to resistant varieties. The recognitions of the reproductive characteristics of the carnation hybrids, object of this work, permitted in a prudent period establish a program of varieties production in Colombia. In this work we used hybrids of three successive generations obtained by autopollination studding the reproductive characteristics. As much as pollen and ovules in the flowers, as well as in the male and female reproductive organs we find a high variability in the number of structures, were the reproductive variability is not bound to the endogamous generation. The pollen quantity is not directly bound with the number of seeds produced, as well as with the number of ovules in the gynoecium. Conversely, the seeds number is related directly with the number of viable ovules by flower. The hybrid fertility expressed in terms of number of pollen grains vs fertilized ovules and produced seeds by flower show that exist a plenty variability in the reproductive conditions in the hybrids, but enough to permit a successful production of seeds.
\end{abstract}

Key words: Carnation, breeding, hybrids, plant reproductive organs.

\footnotetext{
${ }^{1}$ Bióloga, Universidad Militar Nueva Granada, Campus Nueva Granada, Facultad de Ciencias Básicas.

2* Ph.D. Profesor Asociado, Universidad Militar Nueva Granada, Facultad de Ciencias Básicas, Laboratorio de Biotecnología Vegetal, Km 2 vía Cajica-Zipaquirá Colombia. 57+3133752481, juan.filgueira@unimilitar.edu.co
} 


\section{INTRODUCCIÓN}

El clavel comercial pertenece al género Dianthus, familia Caryophyllaceae, especie Dianthus caryophyllus. El clavel actual es derivado de especies ancestrales de D. caryophyllus, y es nativo de Europa Meridional y Asia Occidental (Filgueira, 2011).

Existen principalmente tres eco-tipos de clavel: el Mediterráneo producido entre 1955 y 1980, que crece en la mayor parte de Italia y algunas zonas de Francia, el eco-tipo Sim, derivado de la variedad William Sim y el eco-tipo miniatura o spray, desarrollado en Estados Unidos por Thomson en 1956 (Bilbao y Castro, 1996; Filgueira, 2011).

El clavel es una planta alógama debido a la protandria definida que exhibe, es decir el desarrollo de los gametos masculinos se da primero que el de los femeninos y por tal razón la autofecundación no es un proceso natural. La flor en esta especie se define además como perfecta, hipógina y por la disposición de las estructuras longistila, la cantidad de óvulos y granos de polen puede ser diferente dependiendo de la variedad estudiada, incluso algunas pueden presentar andro-esterilidad (López, 1995; Bilbao y Castro, 1996).

Al ser hipógina se dice que su ovario es súpero, debido a que se encuentra sobre el receptáculo (Jensen y Salisbury, 1994). Para la realización de los cruces en esta especie es pertinente tener en cuenta los diferentes estados de desarrollo de la flor, para así conocer en que posible estado se encuentran los gametos, para realizar la autopolinización en el momento adecuado (Soto et al., 2013).

En este estudio se estudió las características reproductivas de plantas híbridas de tres generaciones sucesivas (F1-F3), producidas por autofecundación en un programa de mejoramiento de clavel, con miras a determinar la viabilidad de la continuidad de las características reproductivas en las generaciones sucesivas, obtenidas por autopolinización y de esta forma garantizar el sostenimiento del programa para obtener variedades comerciales resistentes a parásitos vasculares (Filgueira, 2009; Soto et al., 2012).

\section{MATERIALES Y MÉTODOS}

Material Biológico. En la primera parte de este estudio se utilizaron plantas de clavel provenientes del "Programa de producción de nuevas variedades de clavel de la Universidad Militar Nueva Granada" y correspondían a los híbridos UM146, UM225, UM227, UM226, UM253, UM358 y UM398 de generaciones sucesivas F1, F2 y F3, obtenidas por autopolinización. Los híbridos F1 se obtuvieron por cruce de variedades comerciales susceptibles a parásitos vasculares con variedades silvestres resistentes a parásitos vasculares en los invernaderos de la Facultad de Ciencias Básicas en Cajicá, Cundinamarca.

Se tomaron 100 plantas con 3 a 4 tallos florales por planta mantenidas en condiciones de invernadero. Viabilidad del polen. mediante evaluación de la germinación in vitro(Figura 1a), con una solución de 1:1 deácido bórico (50 ppm) y sacarosa al 5\%.

El polen fue obtenido de flores en estado tres de desarrollo (Filgueira, 2011). Se realizaron evaluaciones por triplicado el resultado por variedad de clavel se estableció observando el tiempo de germinación, tomando como germinado, el momento desde el cual comienza a emerger el tubo polínico hasta su emergencia completa. Cuantificación de las estructuras reproductivas del clavel empleado en la autopolinización y cuantificación de los gametos La determinación del número de óvulos (Figura 1e), se realizó al igual que el conteo del polen en los estambres se hizo bajo el estereoscopio. 


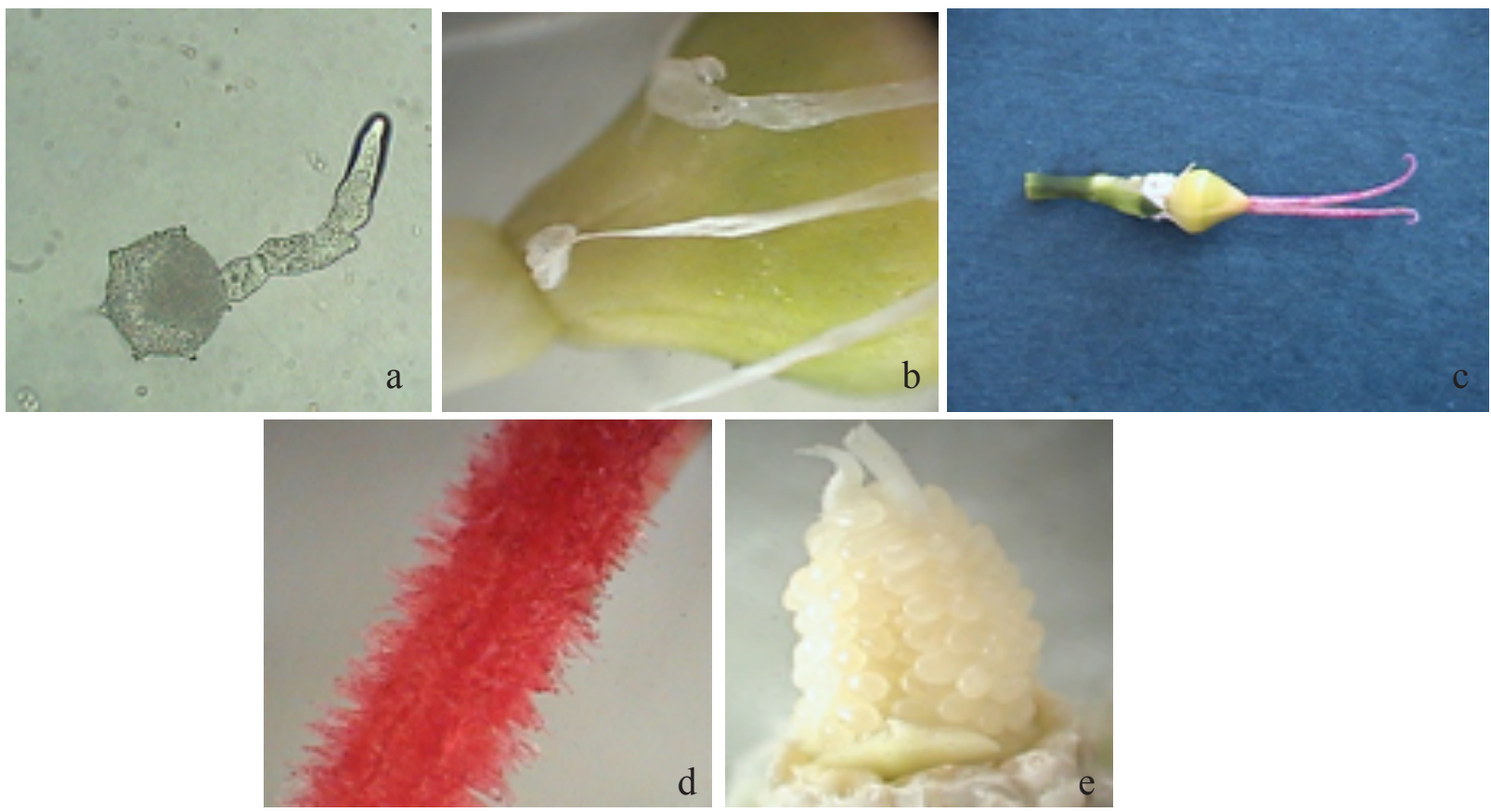

Figura 1. Características de los órganos de reproducción del clavel. a. Grano de polen germinado (40X), donde se observa el tubo polínico. b. Anteras de la variedad comercial Solaris (5X). c. Gineceo con estigmas de la variedad híbrida 225. d. Estigma donde se destaca las papilas turgentes para la fecundación (10X). e. Ovario de la variedad híbrida 253 donde se aprecian los óvulos para ser fecundados (5X).

Para el conteo de los óvulos el receptáculo floral de las flores en estado cuatro fue abierto y se retiraron uno a uno observando su disposición y apariencia. Para el conteo del se emplearon flores en estado tres de desarrollo. Las anteras se separaron de los estambres y el polen se extrajo diluyéndolo en una cantidad fija de agua $(1 \mathrm{ml})$. La cantidad se determinó con un hemocitómetro (Filgueira, 2011). Obtención de semillas.

De cada híbrido o variedad se tomaron 3 tallos por planta (300 tallos). Se realizó la emasculación de la flor en grado 3 de desarrollo y se almacenaron las anteras en cámaras húmedas por no más de 72 horas. El gineceo se cubrió con una bolsa de velo francés hasta determinar el momento adecuado de receptividad del estigma para esparcir el polen. La fecundación se determinó a través de cambios en el tamaño, coloración y textura del ovario. Aproximadamente 6 o 7 semanas después de la polinización se recolectaron las semillas para el correspondiente análisis. Una vez obtenida la semilla se realizó un análisis estadístico en el que se relacionaron los datos cuantitativos obtenidos para las estructuras reproductivas, gametos $y$ variedades con la cantidad de semillas producidas respectivamente. Los datos se analizaron utilizando promedios generales, varianza y desviaciones estándar utilizando el programa Statistics ${ }^{\circledR}$ v2.1. Las gráficas se realizaron en Excel ${ }^{\circledR}$ de Microsoft.

\section{RESULTADOS Y DISCUSIÓN}

La capacidad de producción de semillas de una variedad depende en gran medida de la viabilidad de los granos de polen, pero también dependen de la cantidad de polen por saco polínico en los estambres. Para este caso se observó una alta variabilidad en el número de anteras en los híbridos con valores que oscilan entre 5,6 anteras por flor para el hibrido UM393 y UM253 y 20,4 anteras para el hibrido UM146 
(Tabla 1). Esta variabilidad se encontró de igual forma en la cantidad de granos de polen por antera donde se presentó el menor valor en el híbrido UM393 con un valor promedio de 6200 granos de polen por antera y el mayor valor se observó en UM358 con 34720 granos de polen por estambre en promedio. Para los dos casos tanto para el número de anteras como para la cantidad de polen, las desviaciones estándar son altas, estos valores están de acuerdo con la variabilidad que se espera observar en híbridos de primera y segunda generación (F1 y F2). En cuanto a la variedad comercial, las desviaciones estándar fueron significativamente más bajas, tanto para el número de anteras como para la cantidad de polen en las anteras, y esto está de acuerdo con el hecho que la variedad comercial fue más estable genéticamente. Algunos de los sacos polínicos de los híbridos 227 y 393 se encontraron vacíos, hecho que está relacionado con la baja cantidad de polen por flor, aún más significativo para el caso

Tabla 1. Anteras, polen por anteras, estigmas y óvulos por flor en las variedades hibridas y comercial.

\begin{tabular}{|c|c|c|c|c|}
\hline Variedad & Anteras & Polen & Estigmas & Estigmas \\
\hline UM393 & $5,6 \pm 2,5$ & $6200 \pm 4000$ & $2 \pm 0$ & $121 \pm 20$ \\
\hline UM146 & $20,4 \pm 7,2$ & $23800 \pm 19200$ & $3 \pm 1$ & $157 \pm 18$ \\
\hline UM358 & $8,4 \pm 4,0$ & $34720 \pm 4058$ & $2,8 \pm 1$ & $160 \pm 7$ \\
\hline UM225 & $19,4 \pm 9,1$ & $25140 \pm 5033$ & $2 \pm 0$ & $140 \pm 30$ \\
\hline UM226 & $11,6 \pm 5,5$ & $18976 \pm 2583$ & $2 \pm 0$ & $198 \pm 35$ \\
\hline UM227 & $15 \pm 2,0$ & $12900 \pm 3079$ & $3 \pm 1$ & $233 \pm 52$ \\
\hline UM253 & $5,6 \pm 4,5$ & $32700 \pm 4104$ & $2 \pm 0$ & $137 \pm 10$ \\
\hline Solaris & $11 \pm 1,2$ & $16600 \pm 2480$ & $3,4 \pm 1$ & $143 \pm 24$ \\
\hline
\end{tabular}

relación directa entre el número de óvulos y la cantidad de semillas producidas como era de esperarse, aunque esto no es un parámetro muy marcado (Figura 2.c). Se encontró, además, una relación inversa entre la disminución de semillas y el aumento de la cantidad de polen por flor, hecho que no es fácil de explicar pero que es evidente (Figura 2.a). Esta relación inversa también se observó entre el número de óvulos y la cantidad de polen, una posible explicación a este resultado es la disponibilidad de recursos del hibrido 393, cuyas flores tienen la menor cantidad de óvulos mostrando una relación entre la baja producción de células masculinas y femeninas. Esto muestra una relación genética entre los valores de estas dos variables anatómicas de la flor. No se encontró relación entre la generación deóvulos y la disminución de la producción del polen, hecho que indicaría la perdida de la fertilidad. Esta misma observación es válida también para la producción de semillas que se estableció en este estudio.

El número de estigmas no varió demasiado en los diferentes híbridos y la variedad comercial y no parece estar relacionado con la capacidad reproductiva de la variedad, ya que se encuentran variedades con 2 y 3 estimas y una DS de 1 y un caso excepcional el de la variedad comercial con un valor de 3,4 estigmas por flor en promedio. En este sentido se encuentran híbridos en los cuales varía el número de estigmas y otros donde es muy estable y no cambia, pero nuevamente no se encontró relación entre la variabilidad del número de estigmas y la producción de semillas.

Como en las evaluaciones anteriores para las anteras y el polen, la variabilidad para el numero de óvulos entre las variedades híbridas y entre flores de estos híbridos es alta. El número de óvulos esta entre 120 y 230 por flor en los diferentes híbridos y la variedad comercial. Los valores de la desviación estándar cambian entre 50 y 10 conservando los rangos promedios que se muestran para el numero de órganos sexuales por flor en las diferentes variedades (Tabla 1). Los estigmas se presentaron entre 2 y 3 por flor con una desviación de 1 , un caso excepcional se observó en la variedad comercial con un valor de 3,4 estigmas por flor en promedio.

La germinación de las semillas es un parámetro que no está relacionado con la viabilidad del polen o la cantidad de óvulos o la cantidad de polen en la flor y así fue evidente en este estudio (Tabla 2). Al contrario de lo anterior, existe una 
Tabla 2. Número de semillas y porcentaje de germinación de estas. Se presentan los valores de número de semillas por flor promedio de las variedades hibridas y la variedad comercial, así como los valores de germinación de semillas como el promedio porcentual con la desviación estándar (DS).

\begin{tabular}{|c|c|c|}
\hline Variedad & Anteras & germinación \\
\hline UM393 & 45,2 DS 12 & $34.5 \%$ DS 6,2 \\
\hline UM146 & 33 DS 15 & $22 \%$ DS 3,1 \\
\hline UM358 & 179 DS 37 & $19.5 \%$ DS 4,7 \\
\hline UM225 & 108 DS1 6 & $24.3 \%$ DS 2,8 \\
\hline UM226 & 27,8 DS 8 & $36.5 \%$ DS 5,3 \\
\hline UM227 & 156 DS 24 & $25,4 \%$ DS 1,6 \\
\hline UM253 & 34 DS 14 & $20,5 \%$ DS 3,3 \\
\hline Solaris & 170 DS 33 & $22.3 \%$ DS 3,6 \\
\hline
\end{tabular}

para la formación de las diferentes estructuras. Otro aspecto curioso es que las variedades con la mayor tasa de germinación presentan las cantidades menores de producción de polen.

En general los valores observados en las diferentes estructuras reproductivas se mostraron más estables en la variedad comercial que en los híbridos, hecho que afecta los programas de producción de semillas híbridas y que fue reportado inicialmente por Henao y colaboradores (2007). Estás variaciones muestran híbridos con valores altos de producción de polen en contraste con otros híbridos con valores bajos, pero esto es un indicativo de su inestabilidad en la producción como se ha reportado en otras especies (Ramsey y Vaughton, 2000). El dato más importante y relevante de este estudio en cuanto al interés de producir semillas viables en un programa sostenido de mejoramiento, es que no existe una relación entre las generaciones sucesivas y la disminución de la fertilidad medidas como la cantidad de polen, óvulos o semillas.

Como se indicó anteriormente, híbridos con un bajo número de óvulos presentaron altos valores de germinación y algunos autores relacionan lo anterior en otras especies con disponibilidad de recursos (Kearns y Inuye, 1993). Así mismo, la cantidad de óvulos refleja de alguna forma, la capacidad como progenitor del híbrido, por lo que para fines de un programa de mejoramiento se deben escoger variedades con una baja producción de polen, pero con alta producción de óvulos. Teórica y empíricamente una mayor cantidad de óvulos está asociada a una estrategia reproductiva favorecida evolutivamente en la que dados los recursos disponibles se incrementa la producción máxima de semilla por planta y se hace un uso más eficiente del polen; representado todo, en la formación de mayor número de cigotos (Shreiber et al., 2014; Rosenheim et al., 2015).

Los valores de germinación de los granos de polen oscilaron entre $19,5 \%$ y $36,6 \%$ mostrando que todas las variedades analizadas presentan polen fértil el cual puede interpretarse como alto, ya que como es mencionado en otros trabajos los valores de germinación in vitro suelen ser mucho menores que los valores de germinación natural (Kearns y Inuye, 1993). Igualmente, la polinización manual no es un problema para la producción de semillas en clavel, a pesar de que no hay reportes de polinización natural de la especie y la producción de semillas en estas condiciones, la producción de semilla por fecundación manual es un procedimiento importante en los programas de mejoramiento, incluso algunos trabajos muestran que el rendimiento manual puede ser mayor que el natural (Wilson, 2001) tanto en clavel como en otras especies (Dyaberi et al., 2015). De otra forma los cambios en la productividad de semillas pueden estar relacionados con problemas en la viabilidad de las células de los estigmas como se ha señalado en otras especies (Franklin y Rudd, 2003).

\section{CONCLUSIONES}

Dado que el cultivo del clavel en Colombia es uno de los renglones más importantes de las exportaciones nacionales es necesario que el país produzca nuevas variedades para suplir las 
necesidades de los mercados internacionales, más cuando las variedades producidas en Europa no son resistentes en términos generales a la fusariosis y son muy costosas. En el presente trabajo se evaluaron cuatro generaciones de clavel producidas por técnicas de mejoramiento convencional, en el que se cruzaron variedades comerciales con variedades silvestres y su descendencia se autopolinizo para estabilizar la expresión génica de las principales características agronómicas. No se encontraron efectos adversos debido a la autopolinización, ni en la producción de órganos reproductores de la flor, ni en la producción de semillas viables en las generaciones sucesivas evaluadas.

La hibridación iter-varietal de tan diferentes orígenes, tampoco afecto la producción de células reproductivas en los órganos de reproducción de la planta ni la calidad de la producción de semilla o su viabilidad. Los estudios mostraron que la viabilidad y la cantidad de células reproductivas por flor en los híbridos depende de características genéticas heredadas o transmitidas a las generaciones siguientes y es independiente de si las semillas son producidas por fertilización cruzada o por autofecundación, la mayor parte de estas características está dominada por genes de los cuales no se conoce su actividad ni el tipo de herencia que tienen.

Finalmente, aquí, demostramos como las técnicas de producción de semillas híbridas de diferentes generaciones y producidas por autopolinización, son una alternativa viable en la búsqueda de variabilidad genética y en el análisis de la expresión de diferentes características genéticas deseables desde el punto de vista agronómico dentro de la población y con propósitos económicos.

\section{AGRADECIMIENTOS}

Este trabajo se realizó con el apoyo económico del proyecto de investigación CIAS-2311 de la Universidad Militar Nueva Granada.

\section{REFERENCIAS}

Bilbao, O. y Castro, C. 1995. Estudios preliminares para la obtención de semilla híbrida de clavel estándar bajo las condiciones de la sabana de Bogotá. Trabajo de grado de Ingeniero Agrónomo. Universidad Nacional de Colombia (Bogotá). 39-43; 46; 68-70.

Dyaberi, A., Dhananjaya, M., Kumar, R. and Rao, M. 2015. Floral biology and seed setting in standard carnation (Dianthus caryophyllus). Indian Journal of Agricultural Sciences 85(9): 1175-80.

Filgueira, J. 2009. Estudio y manejo de la pudrición basal producida por hongos del complejo Fusarium en clavel comercial en la Sabana de Bogotá. Asocolflores 72: 53-54.

Filgueira, J. 2011. Experiencias en mejoramiento del clavel (Dianthus caryophyllus). Universidad Militar Nueva Granada $1^{\mathrm{a}}$ Ed. Bogotá Colombia. ISBN 978-958-8403-41-0.

Franklin, V. and Rudd, J. 2003. Signals and targets of the self-incompatibility response in pollen of Papaver rhoeas. Journal of Experimental Botany, 54(380): 141148. https://doi.org/10.1093/jxb/erg001

Henao, L. Clavijo, M., y Filgueira, J. 2007. Capacidad auto reproductiva y morfología floral de algunas lineas experimentales de clavel (Dianthus caryophyllus). Revista Facultad de Ciencias Básicas Universidad Militar Nueva Granada 3(1): 115-125.

Jensen, W. and Salisbury, F. 1994. Botany. Mc Graw-Hill. México. 562-573.

Kearns, C. and Inuye, D. 1993. Techniques for pollination biologist. University Press of Colorado. 36-45;64-75; 90-106;112-122.

López-Torres, M. 1995. Fitomejoramiento. Editorial Trillas. México. 24(93): 141-144.

Ramsey,M.andVaughton, G.2000. Pollenquality limits seed set in Burchardia umbellata. American Journal of Botany 87: 845852. https://doi.org/10.2307/2656892 
Rosenhaim, J., Shreiber, S. and Williams, N. 2016. Does an 'oversupply' of ovules cause pollen limitation? New Phytologist 210: 324-332. https://doi.org/10.1111/nph.13750

Shreiber, S., Rosenhaim, J. Williams, N. and Harder, L. 2015. Evolutionary and ecological consequences of multiscale variation in pollen receipt for seed production. The American Naturalist 185(1). https://doi.org/10.1086/678982

Soto-Sedano, J., Clavijo-Ortiz, M.J. and Filgueira-Duarte, J.J. 2012. Phenotypic evaluation of the resistance in $\mathrm{F} 1$ carnation populations to vascular wilt caused by Fusarium oxysporum f.sp. dianthi. Agronomía Colombiana 30(2): 172-178.
Ramsey,M.andVaughton, G.2000. Pollenquality limits seed set in Burchardia umbellata. American Journal of Botany 87: 845852. https://doi.org/10.2307/2656892

Soto, J., Clavijo, M. and Filgueira, J. 2013. Floral evaluation and reproduction capacity of Dianthus caryophyllus cultivars for a breading program. Acta Horticulturae 1000: 429-432. https://doi. org/10.17660/ActaHortic.2013.1000.59

Wilson, C. 2001. Floral stages, ovule development, and ovule and fruit success in Iris tenax, focusing on var. Gormanii, a taxon with low seed set. American Journal of Botany 88: 22212231. https://doi.org/10.2307/3558384 